\title{
A finite element model of an induction motor considering rotor skew and harmonics
}

\author{
F. T. Oliveira ${ }^{1}$ and M.P. Donsión ${ }^{2}$ \\ ${ }^{1}$ Polytechnic Institute of Leiria, Portugal and INESCC, Portugal \\ e-mail: ftadeu@yahoo.com \\ 2 Department of Electrical Engineering \\ E.T.S.I.I., Vigo University \\ Campus of Lagoas - Marcosende, 36310 Vigo (Spain)
}

\begin{abstract}
Finite element analysis is widely used in engineering, and has for some time been used in modelling the behaviour of an induction motor. Limitations and challenges of this approach will be addressed over a case-study commercial 0,37 kW, 4-pole squirrel-cage induction motor simulated using two-dimensional software FEMM. A few notes on the consideration of rotor skew and harmonic distortion in such a model are also included.
\end{abstract}

\section{Key words}

Finite element analysis; induction motor; harmonics.

\section{Introduction}

FEMM is a finite elements software package for solving low-frequency electromagnetic problems on twodimensional planar and axisymmetric domains. It allows the simulation of linear and nonlinear magnetostatic problems, linear and nonlinear time harmonic magnetic problems, linear electrostatic problems, and steady-state heat flow problems. It can be used in a variety of electromagnetic problems, but has a few limitations when applied to machines with moving parts.

The main purpose of this work is to explore the details of the electromagnetic behaviour of the machine, namely regarding the electromagnetic torque produced in the rotor of the machine.

The results presented in this paper are based upon simulation of the geometric and constructive details of a small, existing commercial induction motor, which was dismantled after having suffered a severe overvoltage.

\section{Case-study problem}

The motor in question is a $0,37 \mathrm{~kW}$, 4-pole, $50 \mathrm{~Hz}$ squirrel-cage induction motor manufactured by Siemens.
It is a standard (IE1) efficiency machine, dismantled after a severe overvoltage.

The dismantled motor was thoroughly measured, and the data was transferred to the software via a CAD drawing. After successfully importing the drawing, the appropriate materials, circuits, definitions and other settings were introduced and checked, in order to be able to obtain the first results.

A planar, time-harmonic magnetic problem (i.e. using a set frequency) was used to represent the machine in question.

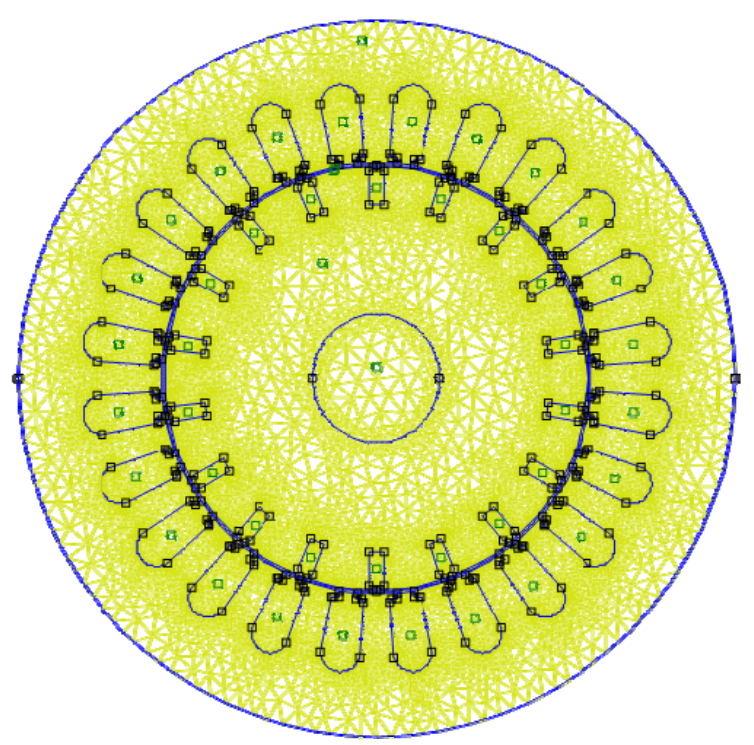

Fig. 1. Drawing of the problem with the triangle mesh

In a planar two-dimensional problem, the circuit is drawn like one of the lamination sheets of the stator and rotor. The third dimension (depth), in this case, is considered 
linearly, and corresponds to the length of the portion of the shaft that is involved by the stator. In our case study, the depth of the problem is $55 \mathrm{~mm}$, as measured in the actual motor.

Any quality results are very much dependent on the correct setting of the materials and circuits. The program is able to deal with laminated non-linear materials, such as the laminated silicon-iron used to build the stator and rotor of this motor.

This construction, of course, helps to limit eddy currents induced in the iron due to the fact that it is an electric conducting material. The laminated stack retains the magnetic permeability of the iron, but, due to insulation between sheets, limits eddy currents along the stator.

The software also allows for eddy current losses to be calculated but, as will be patent further in the paper, those calculated in this case are to be disregarded due to the frequency used in the simulation.

\section{Rotary motion simulation}

One of the main difficulties of using finite element methods with motors is dealing with the fact that the rotor rotates. This is a challenge mostly because software packages are not specifically designed to consider this.

Considering the example presented, the machine is supposedly fed at $50 \mathrm{~Hz}$. The current in each coil would thus be defined and inserted in the simulation problem as a complex number, corresponding to the amplitude and angle of the current in each phase.

However, because the rotor is moving, with a speed which is slightly lower than the synchronous speed, the field induced in the rotor (i.e. "what the rotor sees") is actually variating at a speed which is the slip speed of the rotor. In our example, the nominal speed is $1370 \mathrm{rpm}$ for a 4-pole motor, so the slip is $1500-1370=130$ $\mathrm{rpm}=2.1667 \mathrm{~Hz}$.

Therefore, if we want to have a reasonable perspective of what is happening in the rotor, the simulation will have to be performed at this frequency, which is actually a sort of "trick" in order to accommodate motion.

This, however, does not provide a complete solution to the problem. In reality, the stator coils' currents are not injected at this frequency, but rather at $50 \mathrm{~Hz}$. When simulating the behaviour of the stator, the results obtained for several parameters, including inductance and linkage flux, depend on frequency and will not be accurately calculated. For example, losses and power will be miscalculated.

If, on the other hand, one chose to simulate at a frequency of $50 \mathrm{~Hz}$ (which is accurate for the stator parameters), this would be similar to having a static rotor, such as in a locked-rotor test, performed at nominal current and reduced voltage.

Thus, due to these limitations, it is not possible to produce a model which is accurate in every characteristic of the motor including rotary motion.

\section{Simulation and basic results}

After inserting the problem in the pre-processor and allowing the processor to solve the problem, a postprocessor is available to extract and derive from the solution the relevant quantities.

One of the interesting results is the flux density plot, in which a graphical representation of the distribution of the magnetic field across the transversal cut of the motor.
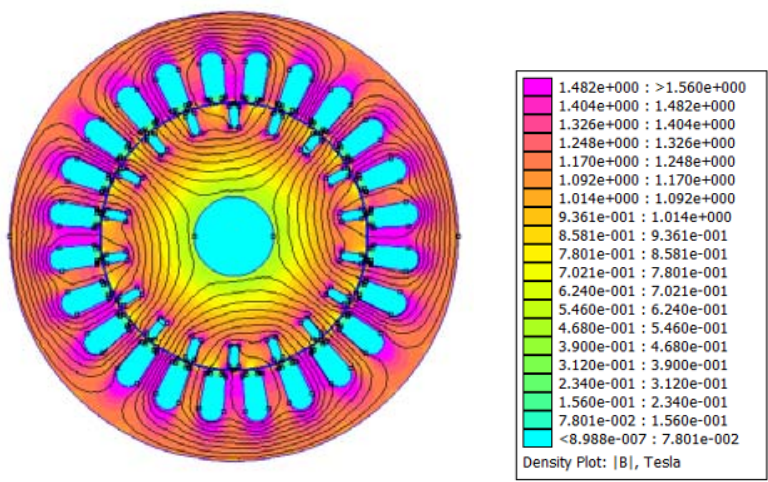

Fig. 2. Flux density $(|\mathrm{B}|[\mathrm{T}])$ in the plane

The contour plot lines outline the four poles, as well as highlighting the difference between slots aligned and not aligned with rotor bars.

Another interesting result is the distribution of the magnetic field across the airgap between stator and rotor. Figure 3 presents the normal component (that which actually crosses the airgap) along the contour of the airgap.

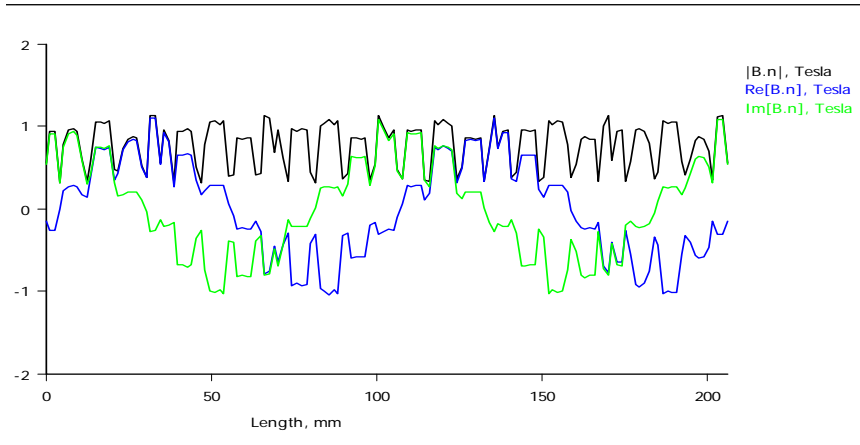

Fig. 3. Plot of flux density (|B| [T]) across the airgap

The rate of change of these lines also stresses the inherent heterogenous nature of the machine. 
Figure 4 shows the area of the rotor selected, and the result from the torque around the centre point calculated from the weighted stress tensor.

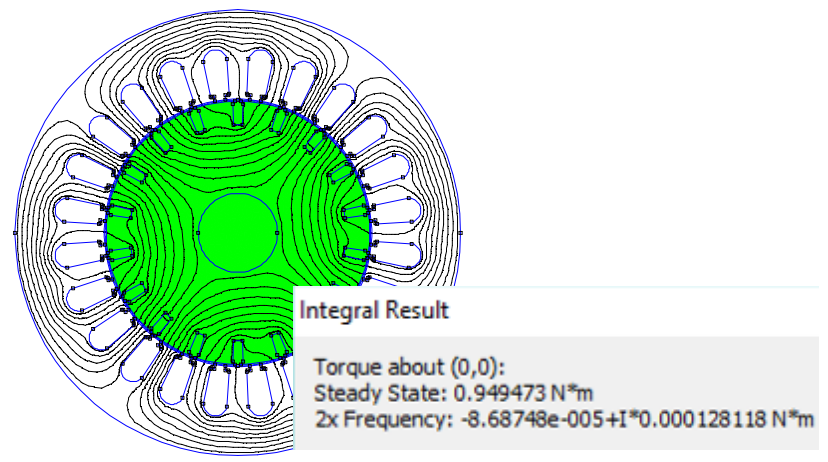

Fig. 4. Rotor torque output

\section{Rotor skew and symmetry considerations}

Looking at the drawing of the stator and rotor transversal cut, it is patent that the stator contains 24 slots in which windings are inserted, and the rotor contains 18 bars (which are, of course, short-circuited at both ends). The angle between two consecutive rotor bars is, therefore, of $20^{\circ}$, while the angle between two consecutive stator slots is $15^{\circ}$.

While the rotor is apparently "homogenous", with rotor bars being all equal, that is not the case with the stator, with slots being filled with different phase windings and polarities.

For this case, in a "minimal" construction, to reduce computational effort, a minimum of a quarter of the stator would have to be simulated (the rest could be obtained through the adequate symmetry boundary conditions), while the stator would require no more than $1 / 18$ of the circle $\left(20^{\circ}\right.$, the minimum period). Problem reduction was dismissed since the complexity of the problem, in present day computers, allows fast simulations.

Since geometry is so important in this type of problem, it is worth noting that the results obtained and presented in the previous chapter correspond to a single relative position of the rotor relative to the stator. Even considering that the rotor can be represented by $20^{\circ}$ out of the whole circle, the rotor bar can occupy different positions along this angle.

These symmetry and rotational considerations are meant to introduce the consideration of the rotor skew issue. When simulating the geometry of a squirrel-cage induction motor, the squirrel-cage bars are actually skewed, so travelling along the depth of the rotor, the different plane cuts will rotate going from one rotor bar "slot" to the next, i.e., with a rotation angle which is the angular difference between adjacent rotor bar "slots".

Different rotor locations, with respect to the stator, provide considerable differences in the electromagnetic behaviour of the set. This means, for example, that the torque value calculated and presented in figure 4 is not an "average" value, but a punctual sample considering that particular rotor-stator alignment.

In order to obtain more insight on this issue, and since the rotor can be fundamentally reduced to a $20^{\circ}$ slice, simulations were conducted with the rotor rotating with respect to the stator, with a rate of $1^{\circ}$, totalling 20 different positions. Considering rotor symmetry, this is equivalent to sweeping the full $360^{\circ}$, with a step of $1^{\circ}$. The results obtained for the torque are shown in figure 5 .

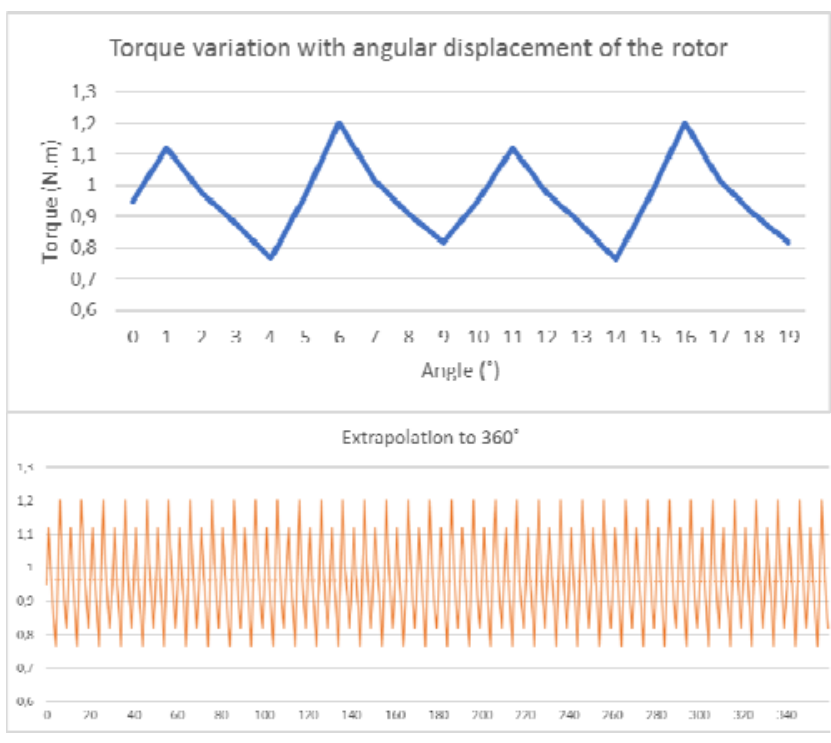

Fig. 5. Rotor torque output variation with the rotor-stator position (simulated values and $360^{\circ}$ extrapolation)

Back to rotor skew, and to this working example, the stator length is of $55 \mathrm{~mm}$. Therefore, if all 20 simulations were condensed and integrated, each one would represent about 2,25 mm of actual depth, improving the accuracy of the representation.

\section{Introduction of harmonics}

Given the time-harmonic proprieties of the problem, and the impossibility to use but one frequency, a possibility to simulate, even if parcially, the effects of harmonic distortion is to run separate sets of simulations at the different harmonic frequencies. Figures 6 to 8 represent the flux density plot of the motor lamination considering a current of $10 \%$ of the nominal current, for the third, fifth and seventh harmonics. 


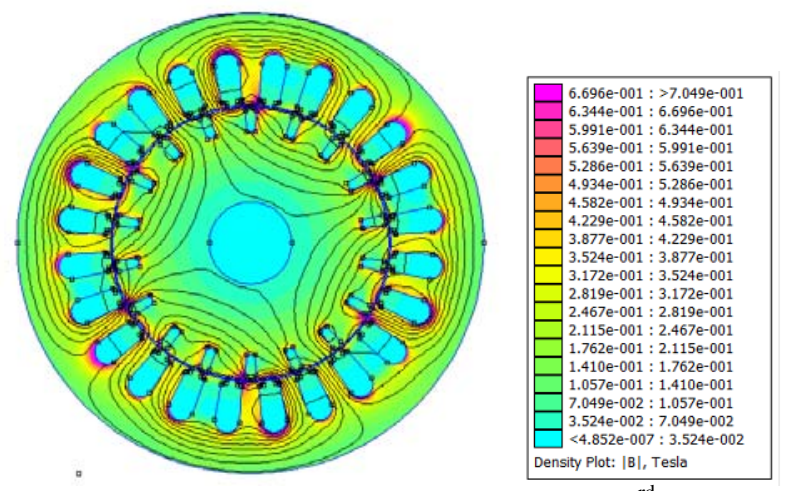

Fig. 6. Flux density for circuit with $10 \%$ current, $3^{\text {rd }}$ harmonic

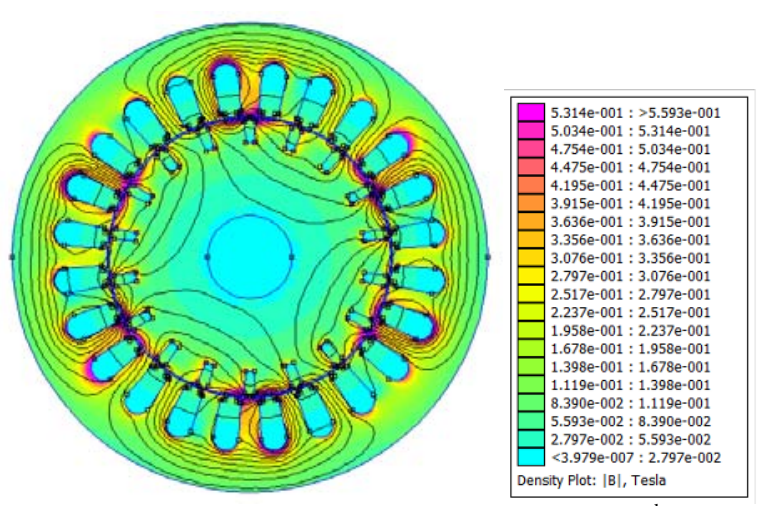

Fig. 7. Flux density for circuit with $10 \%$ current, $5^{\text {rd }}$ harmonic

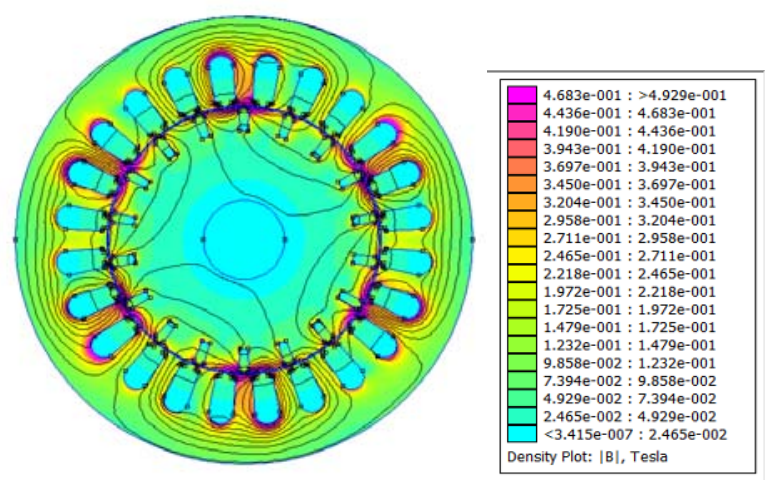

Fig. 8. Flux density for circuit with $10 \%$ current, $7^{\text {rd }}$ harmonic

In order to include the results from simulating the different harmonics, these would have to be considered as "added" to the fundamental component. Considering quantities such as torque, current density, or flux, and with proper care, these could be added to compose the total value.

\section{Conclusions and outlook}

In this paper, the use of finite element analysis, through FEMM software, to simulate the behaviour of an induction motor was explored, providing mixed results.

The simulation results allow a closer insight to the spatial distribution of several electromagnetical quantities, as well as their variation (sensibility) with changes of constructive parameters.

Although much can be explored and obtained through these methods, it is also clear that a number of limitations still hold these methods back, specially when addressing problems with motion, such as those involving motors.

Constructive aspects, both in terms of geometry and properties of materials, are those which are more accurately addressed by this methods.

Future work could see a multi-layer programming of these simulations, building models with multiple "slices" by integrating results space-wise and time-wise. Integration and cross-simultation with other simulation methods and packages could also compensate for the shortcomings of this approach.

\section{References}

[1] D. C. Meeker, Finite Element Method Magnetics, Version 4.2, http://www.femm.info

[2] R. Bargalló, Finite elements for electrical engineering, UPC, 2006

[3] A. Krings, Iron Losses in Electrical Machines - Influence of Material Properties,Manufacturing Processes, and Inverter Operation (PhD thesis), KTH Sweden

[4] N. Bianchi, L. Alberti, Finite element analysis of the induction motor, University of Padua 